Short communication

\title{
Species delineation and hybrid identification using diagnostic nuclear markers for Plectropomus leopardus and Plectropomus maculatus
}

\author{
Song He $\mathrm{He}^{\mathrm{a}, \mathrm{b}, *}$, Hugo B. Harrison ${ }^{\mathrm{c}}$, Michael L. Berumen ${ }^{\mathrm{a}}$ \\ a Red Sea Research Center, Division of Biological and Environmental Science and Engineering, King Abdullah University of Science and Technology, Thuwal, 23955, Saudi \\ Arabia \\ ${ }^{\mathrm{b}}$ State Key Laboratory of Marine Environmental Science, College of Ocean and Earth Sciences, Xiamen University, Xiamen, 361102, Fujian Province, China \\ ${ }^{\mathrm{c}}$ Australian Research Council Centre of Excellence for Coral Reef Studies, James Cook University, Townsville, QLD, 4811, Australia
}

\section{A R T I C L E I N F O}

\section{Handled by J. Viñas}

\section{Keywords:}

Coral trout

Hybridization

Diagnostic markers

Molecular identification

\begin{abstract}
A B S T R A C T
Diagnostic molecular markers are an essential tool in the study of species' ecology and evolution, particularly in closely related and sympatric species. Furthermore, the increasing awareness of wild-hybrids has led to a renewed interest in rapid diagnostic assays. Here, we test the ability of two mitochondrial (Cytb and COI) and two nuclear markers (ETS2 and TMO-4c4) to confidently discriminate purebred $P$. leopardus and $P$. maculatus and their first-generation hybrids. A sample of 48 purebred individuals and 91 interspecific hybrids were used in this study and their delineation confirmed using a set of microsatellite markers. Our results indicate mitochondrial markers could not distinguish even between species but both nuclear markers confidently identified species and first-generation hybrids. However, later-generation hybrids could not always be confidently identified due to ongoing introgression between species. Our findings provide a robust tool to distinguish purebred individuals and interspecific hybrids in a pair of species with an unexpectedly high incidence of hybridization. The quick species discrimination abilities provided by these diagnostic markers are important for stock assessment and recruitment studies of these important fishery species.
\end{abstract}

\section{Introduction}

Molecular markers are widely used in phylogenetic studies to reveal the history of species' evolution and are increasingly used in ecological or management frameworks for species identification. Coral trout (Plectropomus spp., family Serranidae) are commercially important and widely distributed coral reef fishes, and two species commonly occur on inshore and midshelf reefs of the Great Barrier Reef. The bar cheek coral trout, $P$. maculatus (Bloch, 1790), are generally more frequent on inner-shelf reefs, while the common coral trout, $P$. leopardus (Lacepède, 1802), are more abundant on mid- and outer-shelf reefs of the Great Barrier Reef (GBR), suggesting a difference in habitat preferences (Heemstra et al., 1993; Mapstone et al., 1998; Russ et al., 2008). The two species share many morphological and life history characteristics (Ferreira and Russ, 1994, 1992), and adult individuals are most easily identified by their distinctive spot patterns (Fig. 1). Newly settled juveniles of these two species however, can be very difficult to distinguish in the field. Where the two species co-occur in the GBR, they are also known to produce high proportions of viable hybrids (Harrison et al., 2017).

In the marine environment, fish hybridization is not a rare phenomenon, at least 111 natural fish hybrids have been reported, involving more than 173 species (Montanari et al., 2016). Hybridization may thus represent an important evolutionary mechanism in the marine environment, although the evolutionary significance of the process is somewhat debated (Marie et al., 2007; McMillan et al., 1999; Yaakub et al., 2007, 2006). Naturally occurring putative hybrids between $P$. maculatus and $P$. leopardus have been detected from field collections from reefs near Palm and Whitsunday Islands and the Capricorn Bunker Reefs in the GBR (Harrison et al., 2017; van Herwerden et al., 2006, 2002). Newly recruited juveniles of the two species are difficult to distinguish morphologically and adult hybrids may show some intermediate coloration and patterns such as half-elongated spots on the opercula, which may be subtle and are easily overlooked.

Previous molecular work on the genus has had mixed results. van Herwerden et al. (2002) sequenced individuals from four Plectropomus species but found that mitochondrial fragments (using primers L16007 and H00651 for the hypervariable region I of the control region) were not mutually exclusive and could not distinguish $P$. leopardus from $P$. maculatus. However, according to their study, the nuclear oncogene intron (ETS2) sequences provided some preliminary support to distinguish P. leopardus, P. maculatus, and interspecific hybrids. However,

\footnotetext{
* Corresponding author at: State Key Laboratory of Marine Environmental Science, College of Ocean and Earth Sciences, Xiamen University, Xiamen, 361102, Fujian Province, China.

E-mail address: song.he@kaust.edu.sa (S. He).
} 


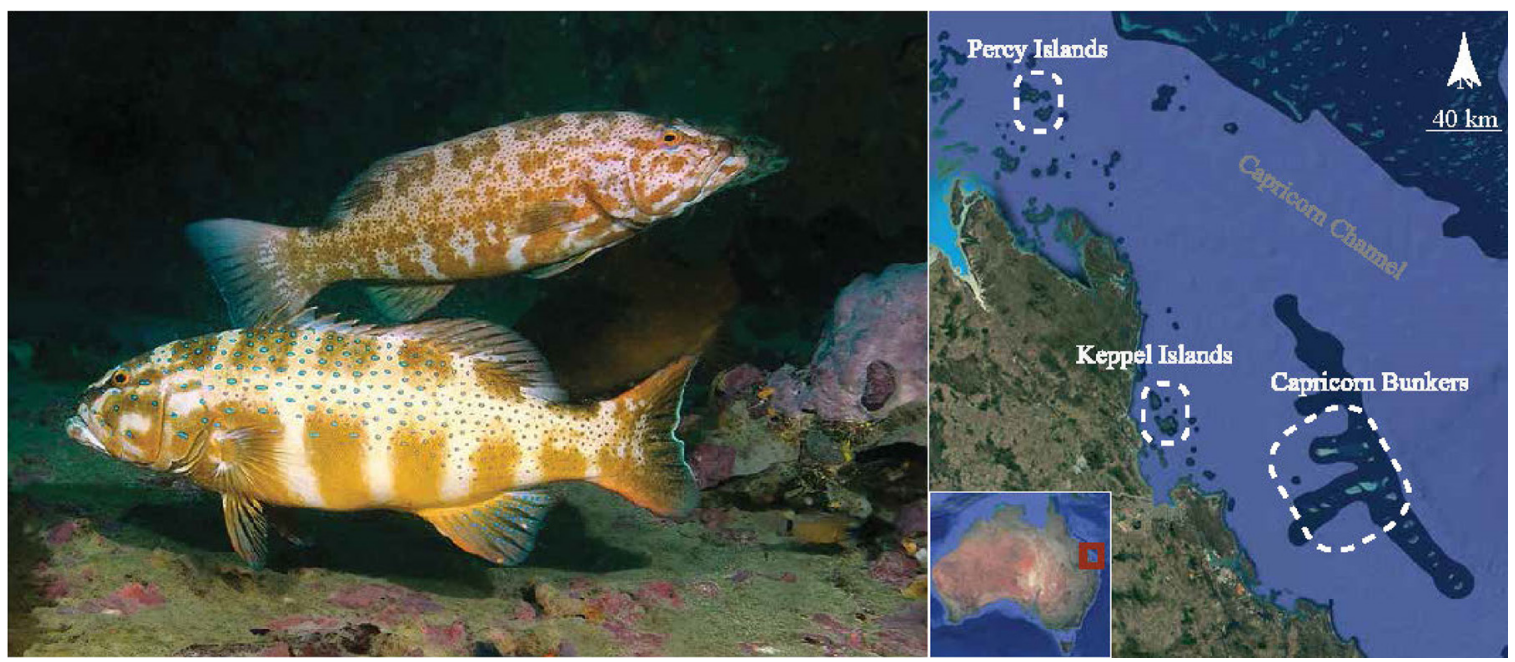

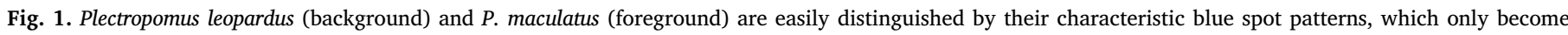

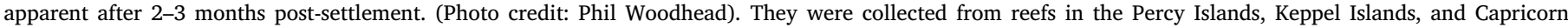

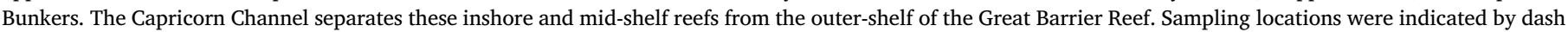

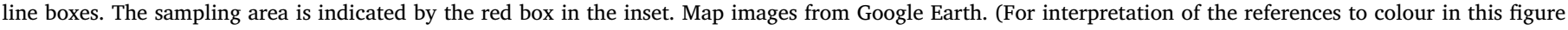
legend, the reader is referred to the web version of this article).

after analyzing additional individuals of $P$. leopardus and $P$. maculatus from the same area, the ETS2 region subsequently failed to delineate the two species (van Herwerden et al., 2006). Instead, a single nuclear microsatellite (locus 7-90 T) appears to have the ability to discriminate the two Plectropomus species, but putative hybrid samples were not tested using this marker (van Herwerden et al., 2006). Still, these markers have been a popular choice for phylogenetic studies for these species (e.g., Ding et al., 2006). Most recently, Ma et al. (2016) confirmed the very close phylogenetic relationship of $P$. leopardus and $P$. maculatus using the ETS2 marker. Subsequently, Harrison et al. (2017) used a panel of 25 microsatellite markers to show that $P$. leopardus and $P$. maculatus hybrids were common in wild populations on the GBR and that contemporary introgression had occurred.

A single diagnostic marker for Plectropomus spp. is important to extend further studies of interspecific hybrids, but also for stock assessment and recruitment studies of these important fishery species. The validation of a simple method to detect and confirm hybrid cases is the first step towards a better general understanding of the processes that underlie hybridization and would enable future studies exploring the associated evolutionary mechanisms involved. Furthermore, identification of juveniles may provide further insights into recruitment patterns and habitat association of newly recruiting individuals. Given the close relationship of $P$. leopardus and $P$. maculatus, the ability to quickly discriminate the two species using a single marker would also have many benefits.

The specific aim of this study was to identify diagnostic markers capable of distinguishing P. leopardus, $P$. maculatus, and their putative hybrids. Ideally, the diagnostic markers should be invariable within a species and have a fixed difference between species. In a previous study, 91 interspecific hybrids were identified from a collection of $2991 P$. leopardus and P. maculatus sampled from reefs in the southern GBR (Fig. 1) (Harrison et al., 2017). These samples were identified using 25 microsatellite markers (Harrison et al., 2014) and were classified as purebred genotypes, first-generation hybrids $(\mathrm{N}=20)$, or second-generation hybrids $(\mathrm{N}=74)$. This previous work provides an ideal sample set containing individuals of known lineage (i.e., purebred of either species or a hybrid of the two) for further genetic investigations. For the present study, two mitochondrial and two nuclear markers were tested to identify potential diagnostic sites in a sample of 48 P. leopardus, $48 P$. maculatus and 91 confirmed hybrids.

\section{Material and methods}

\subsection{Sample collection}

Samples were collected from the Keppel Islands $\left(23^{\circ} 10^{\prime} \mathrm{S}, 150^{\circ} 57^{\prime}\right.$ E), the Percy Islands ( $\left.21^{\circ} 42^{\prime} \mathrm{S}, 150^{\circ} 18^{\prime} \mathrm{E}\right)$, and the Capricorn Bunker reefs $\left(23^{\circ} 25^{\prime} \mathrm{S}, 151^{\circ} 46^{\prime} \mathrm{E}\right)$ in the southern section of the GBR, Australia (Fig. 1). The Keppel and Percy Island groups are chains of high continental islands surrounded by fringing coral reefs, while the CapricornBunker group is comprised of emergent platform reefs located on the outer margin of the continental shelf. Tissue samples for Plectropomus spp. were a subset of those used in Harrison et al. (2017). In brief, these samples were collected from the three previously mentioned locations. All samples were collected between August 2010 and August 2013 under Marine Parks Permit No. G11/3351, Queensland General Fisheries Permit No. 148534, and James Cook University Animal Ethics Permit A1625.

\subsection{DNA analysis}

The analyses presented in this study focused on 91 confirmed hybrids and 48 samples of each of the purebred parent species from Harrison et al. (2017). Ideally, diagnostic markers have no variations within a species, have fixed difference between parent species (i.e., they are mutually exclusive of each other), and have the potential to show hybrids as heterozygous (as an exception to the otherwise mutually exclusive nature of the marker). To investigate potential diagnostic markers, nuclear intron TMO-4c4 and ETS2 fragments were amplified by primers TMO-4C4F/TMO-4C4R and ETS2F/ETS2R (Lyons et al., 1997). To investigate maternal contributions to the hybrids, mitochondrial gene Cytb and COI fragments were amplified by primers Cytb9/Cytb7 (Song et al., 1998) and FishF2/FishR2 (Ward et al., 2005). It is important to note that many other potential diagnostic markers were assessed; including RAG1, RAG2, S7, and BMP4, none of which were suitable for diagnostic purposes (see Appendix 1 in the Supplementary material).

The QIAGEN Multiplex PCR Kit (Qiagen, Hilden, Germany) was used for the polymerized chain reaction. PCR cycling parameters were as follows: initial $95^{\circ} \mathrm{C}$ denaturation for $15 \mathrm{~min}$., followed by 35 cycles of $94^{\circ} \mathrm{C}$ for $45 \mathrm{~s}$., annealing for $60 \mathrm{~s}$. (TMO-4C4F/TMO-4C4R: $60{ }^{\circ} \mathrm{C}$; ETS2F/ ETS2R: $62{ }^{\circ} \mathrm{C}$; Cytb9/Cytb7: $50{ }^{\circ} \mathrm{C}$; FishF2/FishR2: $50{ }^{\circ} \mathrm{C}$ ), and 
$72{ }^{\circ} \mathrm{C}$ for 60 s., and a final elongation step of $72{ }^{\circ} \mathrm{C}$ for $10 \mathrm{~min}$. The PCR products were checked under UV light after running in 1\% agarose gel at $90 \mathrm{~V}$ for $45 \mathrm{~min}$. All PCR products were cleaned by incubating with exonuclease I and FastAP ${ }^{\mathrm{TM}}$ Thermosensitive Alkaline Phosphatase (ExoFAP; USB, Cleveland, OH, USA) at $37^{\circ} \mathrm{C}$ for $60 \mathrm{~min}$., followed by $85^{\circ} \mathrm{C}$ for $15 \mathrm{~min}$. The final products were sequenced in the forward direction with fluorescently labelled dye terminators following the manufacturer's protocols (BigDye, Applied Biosystems Inc., Foster City, CA, USA), and analyzed using an ABI 3130XL Genetic Analyzer (Applied Biosystems).

The sequences were aligned using the program Geneious R8 (Biomatters Ltd., Auckland, New Zealand) and were uploaded to GenBank (Accession No. MG208174 to MG208547). Haplotypes were inferred using the Bayesian analysis PHASE 2.1 (Stephens and Donnelly, 2003) implemented in DnaSP 5.0 (Librado and Rozas, 2009). Each run was performed with a burn-in of 100,000 generations, followed by 200,000 generations. All runs returned consistent results, and were able to phase each haplotype pair with $>90 \%$ posterior probability. Individuals were classified as heterozygotes if their chromatograms exhibited double peaks (Flot and Tillier, 2006), and sequences were delineated using SeqPHASE (Flot, 2010). Median-joining networks showing the relationships among the haplotypes were generated in NETWORK v6.1.3 (Bandelt et al., 1999). The haplowebs (Flot et al., 2010) were derived from the median-joining networks by drawing lines to connect haplotypes that were co-occurring in heterozygous hybrids.

In haplowebs, if all the specimens belong to a single species lineage, the curved connections between haplotypes will conform to HardyWeinberg equilibrium (frequent haplotypes should frequently be found together in heterozygotes). However, if all the specimens belong to two hybridizing species lineages, two haplotype groups conforming to Hardy-Weinberg equilibrium will be observed, but connections between those haplotype groups will be much rarer than if all specimens are conspecific. Rare connections between these two haplotypes would, therefore, represent heterozygous hybrid individuals. Hence, haplowebs can be used to identify hybrids independently and cross-reference the results inferred by other methods.

\section{Results}

\subsection{Mitochondrial DNA}

The length of the alignments was $633 \mathrm{bp}$ for COI fragments and 798 bp for the Cytb and contained 8 and 13 variable sites, which corresponds to 6 and 12 unique haplotypes, respectively. The haplotype diversity was 0.310 for COI and 0.741 for Cytb. For the network-based haploweb, the two Plectropomus clades could not be discriminated by the COI/Cytb haplotypes. The COI/Cytb gene pools of these clades were not mutually exclusive. The mitochondrial haplotypes from the hybrid specimens were indistinguishable from purebred parent species (Fig. 2). With these results, it is not possible to identify the direction of maternal contribution to the hybridization process or even distinguish between pure bred species.

\subsection{Nuclear DNA}

The length of the alignments was $320 \mathrm{bp}$ of the ETS2 fragments and p of the TMO-4c4 fragments and contained 7 and 5 variable sites, which corresponded to 5 and 9 unique haplotypes, respectively. The haplotype diversity was 0.690 for ETS2 and 0.788 for TMO-4c4. For the network based haploweb; the two Plectropomus clades were well-separated by four nucleotide substitutions in ETS2 (Fig. 3) and one insertion in TMO4c4 (Fig. 3). Hence there are five diagnostic sites capable of detecting hybrid individuals. First generation hybrids were determined by heterozygosity at all diagnostic sites. All 17 of the 91 samples were confirmed as F1 hybrids, matching 100\% of the $17 \mathrm{~F} 1$ hybrids identified using microsatellites in Harrison et al. (2017). After the analysis of the
SeqPHASE results, the two haplotypes of the F1 hybrids were identical to parent species (Fig. 3). The F1 hybrid individuals represent exceptions to the otherwise species-specific haplotypes (i.e., the haplotypes are mutually exclusive if the hybrid specimens are excluded) generated by this marker, confirming that these individuals were a hybrid offspring of P. maculatus and P. leopardus.

Harrison et al. (2017) identified 74 individuals as backcrossed hybrids to either parental species. Of these individuals, 41 had homozygous genotypes on one diagnostic marker, confirming their hybrid status and indicating which pure lineage they had backcrossed with (i.e., they remained heterozygous on the other diagnostic marker). Another 12 individuals were heterozygous at all diagnostic sites examined, confirming their hybrid status but not resolving the status further, and 19 were homozygous on both diagnostic markers and matched one of the purebred lineages at all diagnostic sites, precluding our ability to identify these as hybrids using the nuclear marker. Interestingly, two backcrossed individuals were homozygous for both diagnostic markers, but with haplotypes indicating multiple backcrosses to both parent species. Overall, the use of diagnostic markers confirmed all putative F1 hybrids, but only 55 out of 74 backcrossed individuals identified using microsatellite markers. The 19 samples identified as backcrosses by the microsatellite analysis may be F3 or later backcrosses, and as such have the potential to have regained homozygosity in the two diagnostic nuclear markers that tested.

\section{Discussion}

In studies of hybridization, markers that can clearly and reliably separate two purebred lineages are useful as diagnostic markers (Boecklen and Howard, 1997). In principle, ideal markers will show no intraspecific variation (i.e., within each potential parent species) and will show a fixed interspecific difference (i.e., between the two species). Identification of such diagnostic markers requires assessing several potential markers in a suite of samples from both species. The ETS2 and TMo-4c4 markers can be used to distinguish between two closely related Plectropomus species, P. maculatus and P. leopardus. Consistent with previous studies, the two species lineages could not be discriminated by COI and Cytb fragments (van Herwerden et al., 2006), perhaps because of frequent and repeated introgression between these two species (Harrison et al., 2017).

Although van Herwerden et al. (2006) failed to find the ETS2 region useful to discriminate these two species, this region was found informative during this study. Since hybrids are more common than previously appreciated, it is possible that some cryptic hybrid or backcrossed specimens were unknowingly included among their samples and led to the absence of mutual allelic exclusivity on this marker. This would have reduced the apparent discrimination abilities of the nuclear markers. Hybrids need to be removed from the analysis to ensure proper species delimitation (Flot, 2010), although it can be difficult to recognize this problem without a priori information of the purebred status. For samples in our study, identifications were carried out using a large pool of microsatellite data (Harrison et al., 2017) before the samples were used for diagnostic marker investigation. Hence, the interference of hybrids on our ability to detect mutual exclusivity between these two species was not a factor.

The problem of misidentification likely extends beyond identifying diagnostic markers. For example, close examination of numerously published GenBank sequences revealed several questionable cases or likely errors. For example, four ETS2 sequences deposited in GenBank as P. leopardus (DQ666713; DQ666714; KM658838; KM658839) show the diagnostic sequence for P. maculatus. It appears that minor misidentifications such as this may occur frequently. Other examples include a $P$. leopardus sequence (EF517751) deposited as $P$. maculatus. Two ETS2 sequences labelled as P. leopardus (KM658840; KM658841) show heterozygosity at the diagnostic sites, indicating that these are likely hybrid individuals. The error is further propagated by the 


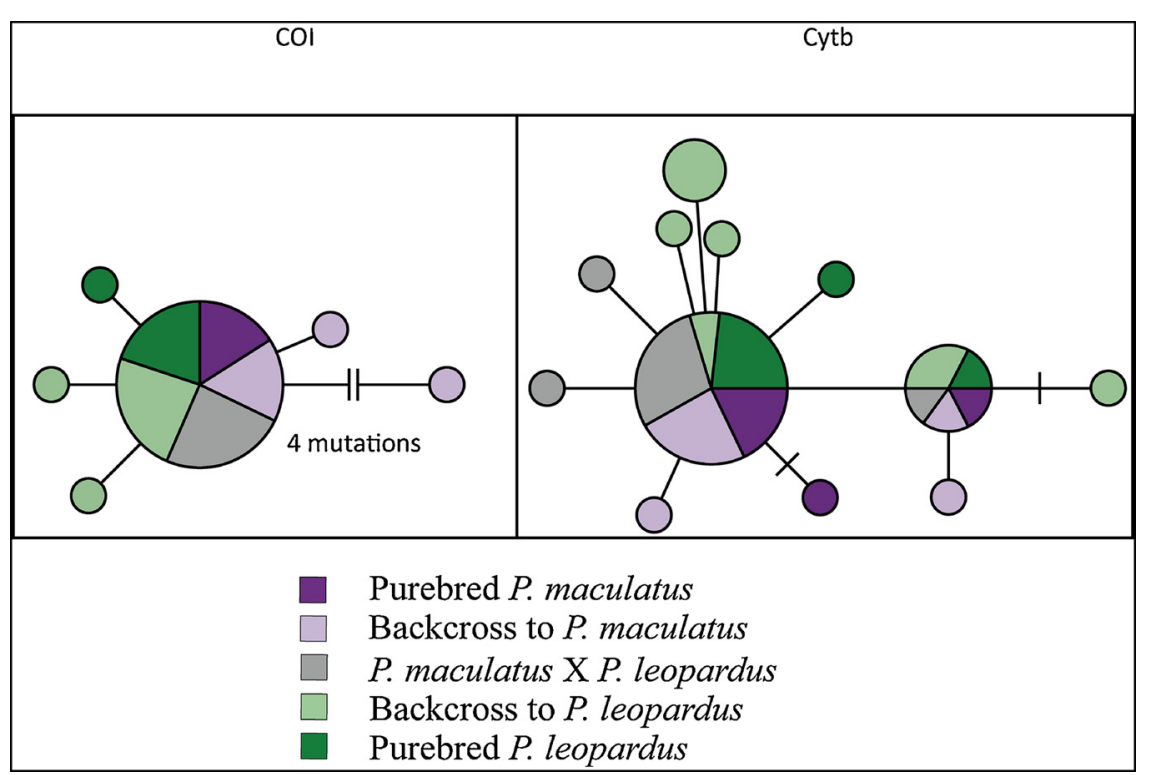

Fig. 2. Phylogenetic relationships of Plectropomus leopardus and P. maculatus, and their hybrids haplotypes represented in Median-joining networks of the COI and Cytb genes. Each circle represents a unique haplotype and size of the circle is proportional to its total frequency. Each branch connecting different circles represents a single nucleotide change and black cross-bars represent an additional nucleotide change. The double bars in the haploweb represent greater than one nucleotide change (as indicated). Colors denote sample species as indicated by the legend. inclusion of some of these sequences in phylogenetic studies. While the outcomes of large phylogenies (such as Ma et al., 2016) are likely only minimally (if at all) impacted by the inclusions of these sequences, more focused examinations of the evolutionary relationship between these two species could be confounded by this issue.

The use of diagnostic markers with heterozygosity in fixed nucleotide positions (i.e., diagnostic sites) between species could be a useful method to identify hybrids from pure bred samples (Bernardi et al., 2013; Coleman et al., 2014; He et al., 2017). Theoretically, 25-30 nuclear markers are needed to produce very high resolution and confidence for documenting interspecific hybrids (Boecklen and Howard, 1997). However, our results suggest that low numbers of nuclear diagnostic markers could confidently identify first-generation hybrids. While 55 of 74 backcrossed individuals were confirmed as hybrids, 19 individuals matched purebred lineages on all diagnostic sites, which precludes our ability to confidently identify later-generation hybrids using these two nuclear markers.

\section{Declarations of interest}

None.

\section{Acknowledgments}

We thank King Abdullah and University of Science and Technology (KAUST) Bioscience Core Laboratory for laboratory support. We acknowledge Jean-François Flot and Tane Sinclair-Taylor for helpful discussions and assistance with figures. Financial support was provided by KAUST baseline research funds to M. L. B. We also thank anonymous reviewers for their constructive comments.

\section{Appendix A. Supplementary data}

Supplementary material related to this article can be found, in the online version, at doi:https://doi.org/10.1016/j.fishres.2018.05.022.

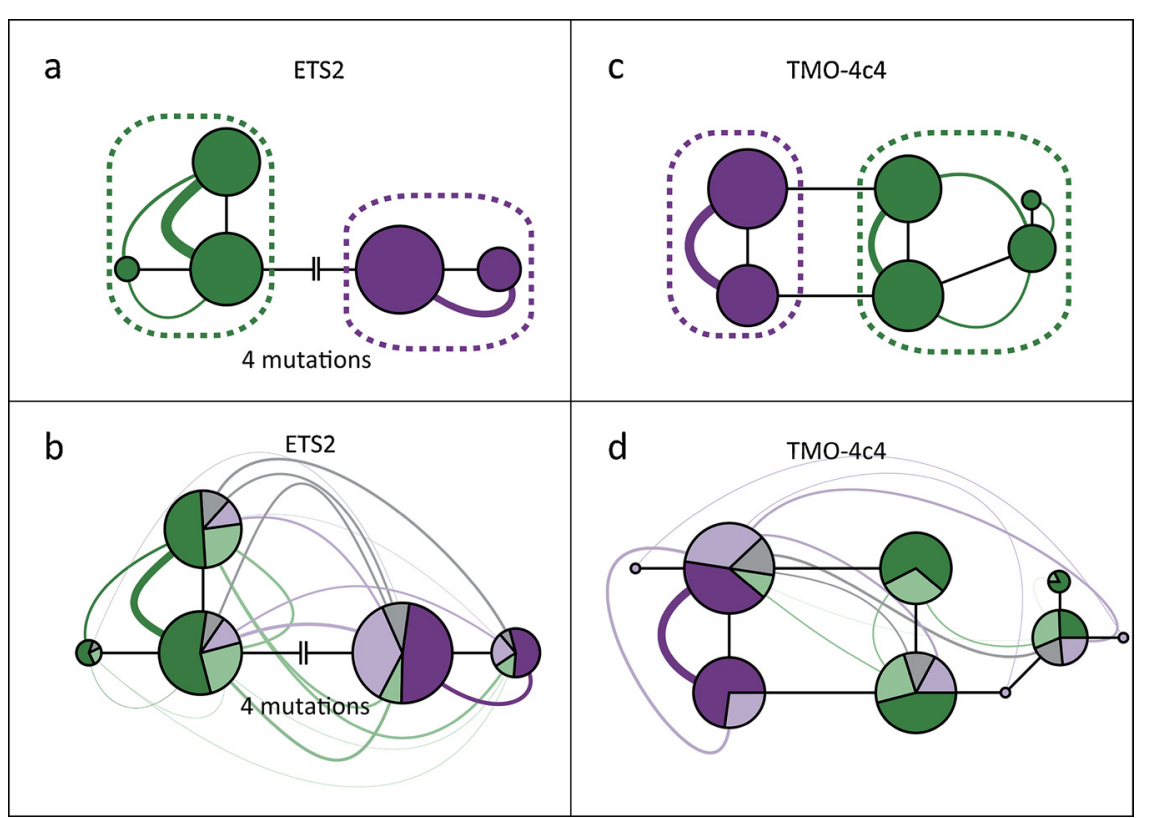

Fig. 3. Phylogenetic relationships of Plectropomus leopardus, $P$. maculatus, and their hybrids haplotypes represented in network-based haplowebs (Flot et al., 2010) of the ETS2 gene (panel a and b) and TMO-4c4 gene (panel c and d). Each circle represents a unique haplotype and size of the circle is proportional to its total frequency. Each branch connecting different circles represents a single nucleotide change and the double bars in the haploweb represent greater than one nucleotide change (as indicated). Curves connecting branches indicate these haplotypes occurring in heterozygous individuals. Dashed line, round corner rectangles represent the gene pool of each purebred species of that gene marker (panel a and c). After including the hybrids, the mutually exclusive status between purebred species vanished (panel $b$ and $d$ ). Colors denote sample species as indicated by the legend in Fig. 2. 


\section{References}

Bandelt, H.-J., Forster, Peter, Röhl, Arne, 1999. Median-joining networks for inferring intraspecific phylogenies. Mol. Biol. Evol. 16, 37-48.

Bernardi, G., Noguchi, R., Anderson, A.B., Floeter, S.R., Ferreira, C.E.L., 2013. Sargo amarelo, a traditionally recognized hybrid between two species of Brazilian reef fishes. Mar. Biodivers. 43, 255-256.

Boecklen, W.J., Howard, D.J., 1997. Genetic analysis of hybrid zones: numbers of markers and power of resolution. Ecology 78, 2611-2616.

Coleman, R.R., Gaither, M.R., Kimokeo, B., Stanton, F.G., Bowen, B.W., Toonen, R.J., 2014. Large-scale introduction of the Indo-Pacific damselfish Abudefduf vaigiensis into Hawaii promotes genetic swamping of the endemic congener A. abdominalis. Mol. Ecol. 23, 5552-5565.

Ding, S., Zhuang, X., Guo, F., Wang, J., Su, Y., Zhang, Q., Li, Q., 2006. Molecular phylogenetic relationships of China Seas groupers based on cytochrome b gene fragment sequences. Sci. China Ser. C 49, 235-242.

Ferreira, B., Russ, G., 1994. Age validation and estimation of growth rate of the coral trout, Plectropomus leopardus, (Lacepede 1802) from Lizard Island, Northern Great Barrier Reef. Fish. Bull. 92, 46-57.

Ferreira, B., Russ, G.R., 1992. Age, growth and mortality of the inshore coarl trout Plectropomus maculatus (Pisces: Serranidae) from the Central Great Barrier Reef, Australia. Mar. Freshw. Res. 43, 1301-1312.

Flot, J.-F., Couloux, A., Tillier, S., 2010. Haplowebs as a graphical tool for delimiting species: a revival of Doyle's" field for recombination" approach and its application to the coral genus Pocillopora in Clipperton. BMC Evol. Biol. 10, 372-386.

Flot, J.-F., Tillier, S., 2006. Molecular phylogeny and systematics of the scleractinian coral genus Pocillopora in Hawaii. Proceedings of the 10th IntErnational Coral Reef Symposium.

Flot, J.F., 2010. SeqPHASE: a web tool for interconverting PHASE input/output files and FASTA sequence alignments. Mol. Ecol. Resour. 10, 162-166.

Harrison, H., Berumen, M., Saenz-Agudelo, P., Salas, E., Williamson, D., Jones, G., 2017. Widespread hybridization and bi-directional introgression in sympatric species of coral reef fish. Mol. Ecol. 1-13. http://dx.doi.org/10.1111/mec.14279.

Harrison, H.B., Feldheim, K.A., Jones, G.P., Ma, K., Mansour, H., Perumal, S., Williamson, D.H., Berumen, M.L., 2014. Validation of microsatellite multiplexes for parentage analysis and species discrimination in two hybridizing species of coral reef fish (Plectropomus spp., Serranidae). Ecol. Evol. 4, 2046-2057.

He, S., Johansen, J.L., Hoey, A.S., Pappas, M.K., Berumen, M.L., 2017. Molecular confirmation of hybridization between Dascyllus reticulatus $\times$ Dascyllus aruanus from the Great barrier Reef. Mar. Biodivers. http://dx.doi.org/10.1007/s12526-12017-1081912528. https://link.springer.com/article/10.1007\%2Fs12526-017-0819-8\#citeas.

Heemstra, P.C., Randall, J.E., FAO, 1993. Species Catalogue Vol. 16 Groupers of the World (Family Serranidae, Subfamily Epinephelinae): An Annotated and Illustrated Catalogue of the Grouper, Rockcod, Hind, Coral Grouper, and Lyretail Species Known to Date. Food and Agriculture Organization of the United Nations, Rome.

Librado, P., Rozas, J., 2009. DnaSP v5: a software for comprehensive analysis of DNA polymorphism data. Bioinformatics 25, 1451-1452.

Lyons, L., Laughlin, T., Copeland, N., Jenkins, N., Womack, J.O., Brien, S., 1997. Comparative anchor tagged sequences (CATS) for integrative mapping of mammalian genomes. Nat. Genet. 15, 47-56.

Ma, K.Y., Craig, M.T., Choat, J.H., van Herwerden, L., 2016. The historical biogeography of groupers: clade diversification patterns and processes. Mol. Phylogenet. Evol. 100, 21-30.

Mapstone, B.D., Ayling, A., Choat, J., 1998. Habitat, Cross Shelf and Regional Patterns in the Distributions and Abundances of Some Coral Reef Organisms on the Northern Great Barrier Reef, With Comment on the Implications for Future Monitoring. Great Barrier Reef Marine Park Authority, Townsville, Australia.

Marie, A., Van Herwerden, L., Choat, J., Hobbs, J.A., 2007. Hybridization of reef fishes at the Indo-Pacific biogeographic barrier: a case study. Coral Reefs 26, 841-850.

McMillan, W.O., Weigt, L.A., Palumbi, S.R., 1999. Color pattern evolution, assortative mating, and genetic differentiation in brightly colored butterflyfishes (Chaetodontidae). Evolution 53, 247-260.

Montanari, S.R., Hobbs, J.-P.A., Pratchett, M.S., van Herwerden, L., 2016. The importance of ecological and behavioural data in studies of hybridisation among marine fishes. Rev. Fish Biol Fish. 26, 181-198.

Russ, G.R., Cheal, A.J., Dolman, A.M., Emslie, M.J., Evans, R.D., Miller, I., Sweatman, H., Williamson, D.H., 2008. Rapid increase in fish numbers follows creation of world's largest marine reserve network. Curr. Biol. 18, R514-R515.

Song, C.B., Near, T.J., Page, L.M., 1998. Phylogenetic relations among percid fishes as inferred from mitochondrial cytochromebDNA sequence data. Mol. Phylogenet. Evol. 10, 343-353.

Stephens, M., Donnelly, P., 2003. A comparison of bayesian methods for haplotype reconstruction from population genotype data. Am. J. Hum. Genet. 73, 1162-1169.

van Herwerden, L., Choat, J., Dudgeon, C., Carlos, G., Newman, S., Frisch, A., Van Oppen, M., 2006. Contrasting patterns of genetic structure in two species of the coral trout Plectropomus (Serranidae) from east and west Australia: introgressive hybridisation or ancestral polymorphisms. Mol. Phylogenet. Evol. 41, 420-435.

van Herwerden, L., Davies, C., Choat, J., 2002. Phylogenetic and evolutionary perspectives of the Indo-Pacific grouper Plectropomus species on the Great Barrier Reef, Australia. J. Fish Biol. 60, 1591-1596.

Ward, R.D., Zemlak, T.S., Innes, B.H., Last, P.R., Hebert, P.D., 2005. DNA barcoding Australia's fish species. Phil. Trans. R. Soc. B: Biol. Sci. 360, 1847-1857.

Yaakub, S., Bellwood, D., Van Herwerden, L., 2007. A rare hybridization event in two common Caribbean wrasses (genus Halichoeres; family Labridae). Coral Reefs 26, 597-602.

Yaakub, S.M., Bellwood, D.R., van Herwerden, L., Walsh, F.M., 2006. Hybridization in coral reef fishes: introgression and bi-directional gene exchange in Thalassoma (family Labridae). Mol. Phylogenet. Evol. 40, 84-100. 